\title{
A Moving Frame Trajectory Tracking Method of a Flying-Wing UAV Using the Differential Geometry
}

\author{
Yi Zhu, Xin Chen, and Chuntao Li \\ College of Automation Engineering, Nanjing University of Aeronautics and Astronautics, Nanjing 210016, China \\ Correspondence should be addressed to Yi Zhu; zhuyi73@126.com
}

Received 27 July 2016; Accepted 19 October 2016

Academic Editor: Mahmut Reyhanoglu

Copyright ( 2016 Yi Zhu et al. This is an open access article distributed under the Creative Commons Attribution License, which permits unrestricted use, distribution, and reproduction in any medium, provided the original work is properly cited.

\begin{abstract}
The problem of UAV trajectory tracking is a difficult issue for scholars and engineers, especially when the target curve is a complex curve in the three-dimensional space. In this paper, the coordinate frames during the tracking process are transformed to improve the tracking result. Firstly, the basic concepts of the moving frame are given. Secondly the transfer principles of various moving frames are formulated and the Bishop frame is selected as a final choice for its flexibility. Thirdly, the detailed dynamic equations of the moving frame tracking method are formulated. In simulation, a moving frame of an elliptic cylinder helix is formulated precisely. Then, the devised tracking method on the basis of the dynamic equations is tested in a complete flight control system with 6 DOF nonlinear equations of the UAV. The simulation result shows a satisfactory trajectory tracking performance so that the effectiveness and efficiency of the devised tracking method is proved.
\end{abstract}

\section{Introduction}

The main difficulties of a UAV trajectory tracking issue differing from the ordinary tracking problems lie in the strict real-time requirement and the complexity of 3-dimensional curves. Some literature only attempts to deal with the reduced situations such as the "path following" problem without a constrained time parameter [1-3] or the simplified target curves in 2D plane [4-6]. What is more, different types of curves usually call for different guidance strategies. In contrast, the moving frame tracking method is employed to give a more universal solution for various kinds of complex curves in the 3D space.

There are two procedures of the moving frame tracking method.

The first procedure of the moving frame tracking method is to form a moving frame of the given target curve. A moving frame is a moving coordinate axis system with its three axes being the curve's tangent vector, principal normal vector, and secondary normal vector. There are many ways to obtain a moving frame of a given curve [7, 8]. The Bishop frame which is also called the parallel frame is the most famous one $[9,10]$. In recent years, papers tend to concentrate on the improvements of the Bishop frame [11, 12]. In this paper by comparisons of the Frenet frame and the type 1 Bishop frames, we can see some insights of forming a moving frame and find out the reason why the Bishop frame is suitable for solving a UAV trajectory problem.

The second procedure of the moving frame tracking method is to formulate the dynamic error equations of the whole tracking process. In this procedure a quite unusual tracking process is presented. We no longer need to care about the individual shape of the target curve $[13,14]$. That is to say, whether the target curve is a straight line, a circle, or an arc is unimportant. Instead, the moving frame of the curve with its tangent vector, principal normal vector, and secondary normal vector is the objectives to follow. Some knowledge of the theoretical mechanics about the motions of rigid bodies is necessary. In essence, the objective dynamic error equations are the result of a series of coordinate transformations and vector operations $[15,16]$. In this procedure, the final aim is to obtain the kinematics errors in the moving frame of the target curve rather than the inertial coordinate frame.

The previous papers treating the trajectory tracking problem with the moving frame method are not sufficient. The reason is that the moving frame tracking method is very interdisciplinary for the theorists of guidance, navigation, and control. However, the analysis of geometrical 


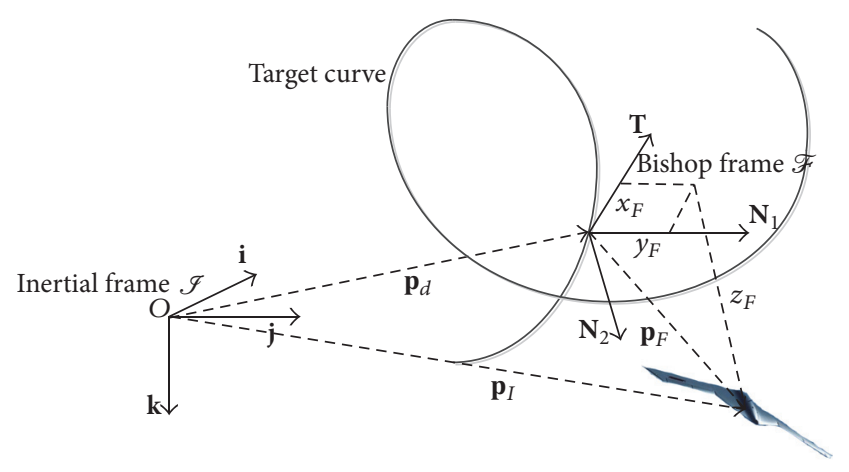

FIGURE 1: Illustration of the moving frame tracking method.

properties of curves actually plays an important role in practical applications. Thus, the moving frame tracking is an effective and practical method for the UAV 3D trajectory tracking problem. In the previous literature some trials have been made to apply this tracking method to the tiny UAVs or quadrotors [15, 17]. Some other sources of literature are concerned about the mathematical features of this tracking method [18, 19]. All these sources of literature tackle the particle models of the microaircrafts with very low flight speed. For example, in literature [15], the velocity of the aircraft is $0.4 \mathrm{~m} / \mathrm{s}$. In this paper a much larger and heavier UAV is used and its velocity is about $200 \mathrm{~m} / \mathrm{s}$ so that the situation is different. Also, it is well known that when a particle model is used, the angular motions inside the rigid body are omitted. So another distinction with the previous literature is that in this paper a 6 DOF UAV model is employed instead of a particle model.

\section{Formulation of the Moving Frame Tracking Method}

2.1. General Illustration. The core idea of the moving frame tracking method lies in the conception that the position errors are resolved in the moving coordinate frame of the target curve instead of the inertial frame in ordinary tracking methods. In this method the time constraint is well satisfied since the target curve is formulated with a time parameter $t$. The primary aim is aligning the heading direction to the tangent vector of the target curve with almost no position deviation. The principle of the moving frame tracking method is illustrated in Figure 1.

It can be seen from Figure 1 that the aircraft used in this paper is a UAV with flying-wing configuration. The target curve, that is, the target trajectory to track, is an elliptic cylinder helix. There are five coordinate frames that need to be introduced which are the inertial frame $\{\mathscr{I}\}$, the body frame $\{\mathscr{B}\}$, the wind frame $\{\mathscr{W}\}$, and the curve frame $\{\mathscr{F}\}$. The inertial frame describes the physical positions on the earth. The body frame is a frame attached to the body of the aircraft. The wind frame is a speed frame with its $x$-axis aligned with the true airspeed of the aircraft, while the other two axes are orthogonally decomposed in the inertial frame.
The curve frame is a moving frame (as the Frenet frame or Bishop frame) of the target trajectory.

2.2. Forming a Moving Frame. By mathematical ways, it is implementable to form a moving frame of a 3D Curve. In the early stages of classical differential geometry, the Frenet frame was adopted to formulate a curve in the three-dimensional Euclidean space $E^{3}$. Let $\mathbf{T}, \mathbf{N}$, and $\mathbf{B}$ represent the tangent vector, principal normal vector, and secondary normal vector, respectively. For all the curves in $E^{3}$, by transforming the time parameter $t$ into an arc length parameter $s$, the Frenet frame of a curve is defined by

$$
\begin{gathered}
\frac{d \mathbf{r}}{d s}=\mathbf{T}, \\
{\left[\begin{array}{c}
\frac{d \mathbf{T}}{d s} \\
\frac{d \mathbf{N}}{d s} \\
\frac{d \mathbf{B}}{d s}
\end{array}\right]=\left[\begin{array}{c}
\kappa \mathbf{N} \\
-\kappa \mathbf{T}+\tau \mathbf{B} \\
-\tau \mathbf{N}
\end{array}\right]=\left[\begin{array}{ccc}
0 & \kappa & 0 \\
-\kappa & 0 & \tau \\
0 & -\tau & 0
\end{array}\right]\left[\begin{array}{c}
\mathbf{T} \\
\mathbf{N} \\
\mathbf{B}
\end{array}\right],}
\end{gathered}
$$

where $\kappa$ and $\tau$ are curvature and torsion of the Frenet frame, defined by

$$
\begin{aligned}
\kappa & =\left|\frac{d \mathbf{T}}{d s}\right|, \\
\tau & =-\mathbf{B}^{\prime} \cdot \mathbf{N} .
\end{aligned}
$$

Today the Frenet frame has been improved to become the Bishop frame. The type 1 Bishop frame is defined by

$$
\begin{aligned}
{\left[\begin{array}{c}
\frac{d \mathbf{T}}{d s} \\
\frac{d \mathbf{N}_{1}}{d s} \\
\frac{d \mathbf{N}_{2}}{d s}
\end{array}\right]=} & {\left[\begin{array}{c}
k_{1}(s) \mathbf{N}_{1}+k_{2}(s) \mathbf{N}_{2} \\
-k_{1}(s) \mathbf{T} \\
-k_{2}(s) \mathbf{T}
\end{array}\right] } \\
= & {\left[\begin{array}{ccc}
0 & k_{1}(s) & k_{2}(s) \\
-k_{1}(s) & 0 & 0 \\
-k_{2}(s) & 0 & 0
\end{array}\right]\left[\begin{array}{c}
\mathbf{T} \\
\mathbf{N}_{1} \\
\mathbf{N}_{2}
\end{array}\right] . }
\end{aligned}
$$

The relationship between the Frenet frame and type 1 Bishop frame is described as follows:

$$
\begin{aligned}
& \mathbf{T}=\mathbf{T}, \\
& \mathbf{N}=\cos \theta_{F} \mathbf{N}_{1}+\sin \theta_{F} \mathbf{N}_{2}, \\
& \mathbf{B}=-\sin \theta_{F} \mathbf{N}_{1}+\cos \theta_{F} \mathbf{N}_{2},
\end{aligned}
$$

where

$$
\begin{gathered}
\theta_{F}(s)=\arctan \frac{k_{2}}{k_{1}} . \\
\tau(s)=\frac{d \theta(s)}{d s}, \\
\kappa(s)=\sqrt{k_{1}^{2}+k_{2}^{2}} .
\end{gathered}
$$


The type 1 Bishop curvatures are defined by

$$
\begin{aligned}
& k_{1}=\kappa \cos \theta_{F}, \\
& k_{2}=\kappa \sin \theta_{F} .
\end{aligned}
$$

From the definition of the Bishop frame and the relationship between it and the Frenet frame we can see that, roughly speaking, the Bishop frame is a frame which has the same original point with the Frenet frame and aligns one of its axes with one of the axes of the Frenet frame. Then the rest of the two axes of the Bishop frame rotate at a certain angle $\theta_{F}$ in the normal plane of the first axis. Obviously, a new generated Bishop frame is always isomorphic to the Frenet frame in $E^{3}$.

2.3. Dynamic Errors Equations of Moving Frame Tracking. Let the inertial frame be defined by $\{\mathscr{I}\}=\{O: \mathbf{i}, \mathbf{j}, \mathbf{k}\}$ with axis $\mathbf{i}$ pointing to the north, axis $\mathbf{j}$ pointing to the east, and axis $\mathbf{k}$ pointing vertically to the center of the earth. So the frame $\{\mathscr{I}\}$ is a NED (North-Easy-Down) frame. Suppose the target curve with an arc length parameter is

$$
\mathbf{P}_{d}(s)=\left[\begin{array}{lll}
P_{d x}(s) & P_{d y}(s) & P_{d z}(s)
\end{array}\right]^{\mathrm{T}} .
$$

Then the trajectory tracking problem is presented as follows: for a target curve $\mathbf{P}_{d}$ with its moving frame and an aircraft model, a controller needs to be designed to force the aircraft to fly along the target curve by means of minimizing the position error vector which is resolved in the moving frame.

Assuming the Bishop frame of the target curve is defined by $\{\mathscr{F}\}=\left\{\mathbf{T}, \mathbf{N}_{1}, \mathbf{N}_{2}\right\}$, according to the definition of the frame $\{\mathscr{W}\}$, the airspeed $V$ equals ground speed when there is no wind disturbance. Thus the speed vector from frame $\{\mathscr{W}\}$ to $\{\mathscr{I}\}$ resolved in frame $\{\mathscr{W}\}$ is represented as $\left.\mathbf{v}_{W 2 I}\right|_{W}=$ $\left[\begin{array}{lll}V & 0 & 0\end{array}\right]$.

Let $\mathbf{p}_{I}=\left[\begin{array}{lll}x & y & z\end{array}\right]^{\mathrm{T}}$ be the vector from the origin of the frame $\{\mathscr{I}\}$ pointing to the aircraft's center of mass resolved in $\{\mathscr{I}\}$ and $\mathbf{p}_{F}=\left[\begin{array}{lll}x_{F} & y_{F} & z_{F}\end{array}\right]^{\mathrm{T}}$ be the vector from the origin of the frame $\{\mathscr{F}\}$ pointing to the aircraft's center of mass resolved in $\{\mathscr{F}\}$. The angular velocity vector of the body frame $\{\mathscr{B}\}$ rotating around $\{\mathscr{I}\}$ and resolved in frame $\{\mathscr{F}\}$ is presented as

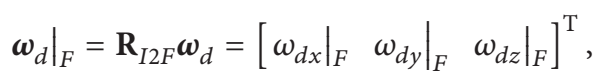

where $\mathbf{R}_{I 2 F} \in \mathrm{SO}(3)$ is the rotation matrix from $\{\mathscr{I}\}$ to $\{\mathscr{F}\}$. $\mathrm{SO}(3)$ is the 3-dimensional special orthogonal group. Also, the matrix $\mathbf{R}_{F 2 I}$ represents the rotation matrix from the frame $\{\mathscr{F}\}$ to the inertial frame. Then, in the light of the kinematics of rigid bodies, (9) holds.

$$
\mathbf{R}_{F 2 I}=\left[\mathbf{T}, \mathbf{N}_{1}, \mathbf{N}_{2}\right] .
$$

The condition is just the same with the other frames, which is no longer repeated here. So formula (9) is an instance of showing a general rule of the coordinate transformation with unitary frames in $E^{3}$.

To be more precise about the meaning and resolving method of the angular velocity vector just mentioned above, some discussions of the Darboux vector are given in the following. The Darboux vector is named after Gaston Darboux who discovered it. It is also called angular momentum vector, because it is directly proportional to angular momentum. For a rigid body moving absolutely along a general curve which is expressed by a moving frame, its motions can be divided into two kinds of motions as the translational motion and the rotational motion. The rotational motion is formulated by the Darboux vector $\boldsymbol{\omega}$ which is a composition of three axes' areal velocity components.

$$
\omega=\omega_{\tilde{\mathrm{i}}}+\omega_{\tilde{\mathrm{j}}}+\omega_{\widetilde{\mathrm{k}}}
$$

where $\tilde{\mathbf{i}}, \tilde{\mathbf{j}}, \widetilde{\mathbf{k}}$ represent axes vectors of a moving frame. Each axis vector rotates around a point. When this moving frame is a unitary frame, each axis areal velocity is defined by

$$
\begin{aligned}
& \omega_{\tilde{\mathbf{i}}}=\lim _{\Delta t \rightarrow 0} \frac{\tilde{\mathbf{i}}(t) \times \tilde{\mathbf{i}}(t+\Delta t)}{2 \Delta t}=\frac{\tilde{\mathbf{i}}(t) \times \widetilde{\mathbf{i}}^{\prime}(t)}{2}, \\
& \omega_{\tilde{\mathbf{j}}}=\lim _{\Delta t \rightarrow 0} \frac{\tilde{\mathbf{j}}(t) \times \tilde{\mathbf{j}}(t+\Delta t)}{2 \Delta t}=\frac{\tilde{\mathbf{j}}(t) \times \widetilde{\mathbf{j}}^{\prime}(t)}{2}, \\
& \omega_{\widetilde{\mathbf{k}}}=\lim _{\Delta t \rightarrow 0} \frac{\widetilde{\mathbf{k}}(t) \times \widetilde{\mathbf{k}}(t+\Delta t)}{2 \Delta t}=\frac{\widetilde{\mathbf{k}}(t) \times \widetilde{\mathbf{k}}^{\prime}(t)}{2} .
\end{aligned}
$$

Taking the Frenet frame, for instance, with $\tilde{\mathbf{i}}, \tilde{\mathbf{j}}$, and $\widetilde{\mathbf{k}}$ replaced by $\mathbf{T}, \mathbf{N}$, and $\mathbf{B}$, one has

$$
\begin{aligned}
& \boldsymbol{\omega}_{\mathrm{T}}=\frac{\mathbf{T}(t) \times \mathbf{T}^{\prime}(t)}{2}=\frac{\kappa \mathbf{B}}{2}, \\
& \boldsymbol{\omega}_{\mathbf{N}}=\frac{\mathbf{N}(t) \times \mathbf{N}^{\prime}(t)}{2}=\frac{\tau \mathbf{T}+\kappa \mathbf{B}}{2}, \\
& \boldsymbol{\omega}_{\mathbf{B}}=\frac{\mathbf{B}(t) \times \mathbf{B}^{\prime}(t)}{2}=\frac{\tau \mathbf{T}}{2}, \\
& \boldsymbol{\omega}=\boldsymbol{\omega}_{\mathrm{T}}+\boldsymbol{\omega}_{\mathbf{N}}+\boldsymbol{\omega}_{\mathbf{B}}=\tau \mathbf{T}+\kappa \mathbf{B} .
\end{aligned}
$$

It is easy to see that the vector $\boldsymbol{\omega}$ satisfies the equations

$$
\left[\begin{array}{l}
\frac{d \mathbf{T}}{d t} \\
\frac{d \mathbf{N}}{d t} \\
\frac{d \mathbf{B}}{d t}
\end{array}\right]=\left[\begin{array}{l}
\boldsymbol{\omega} \times \mathbf{T} \\
\boldsymbol{\omega} \times \mathbf{N} \\
\boldsymbol{\omega} \times \mathbf{B}
\end{array}\right] .
$$

Then concerning the Bishop frame, its Darboux vector is

$$
\begin{aligned}
\boldsymbol{\omega}_{\mathrm{T}} & =\frac{\mathbf{T} \times \mathbf{T}}{2}=\frac{1}{2} \mathbf{T} \times\left(k_{1} \mathbf{N}_{1}+k_{2} \mathbf{N}_{2}\right) \\
& =\frac{1}{2} k_{1} \mathbf{N}_{2}-\frac{1}{2} k_{2} \mathbf{N}_{1}, \\
\boldsymbol{\omega}_{\mathbf{N}_{1}} & =\frac{\mathbf{N}_{1} \times \mathbf{N}_{1}^{\prime}}{2}=\mathbf{N}_{1} \times\left(-\frac{1}{2} k_{1}\right) \mathbf{T}=\frac{1}{2} k_{1} \mathbf{N}_{2}, \\
\boldsymbol{\omega}_{\mathbf{N}_{2}} & =\frac{\mathbf{N}_{2} \times \mathbf{N}_{2}^{\prime}}{2}=\mathbf{N}_{2} \times\left(-\frac{1}{2} k_{2}\right) \mathbf{T}=-\frac{1}{2} k_{2} \mathbf{N}_{1}, \\
\boldsymbol{\omega} & =\boldsymbol{\omega}_{\mathrm{T}}+\boldsymbol{\omega}_{\mathbf{N}_{1}}+\boldsymbol{\omega}_{\mathbf{N}_{2}}=k_{1} \mathbf{N}_{2}-k_{2} \mathbf{N}_{1} .
\end{aligned}
$$


When the moving frame is parameterized by a time parameter $t$, the Darboux vector of the Bishop frame and resolved in the Bishop frame is

$$
\boldsymbol{\omega}_{\text {Darboux } \mid \text { Bishop }}=\left[\begin{array}{llll}
0 & -k_{2}(s) \dot{s} & k_{1}(s) \dot{s}
\end{array}\right]^{\mathrm{T}},
$$

where $s$ is the arc length parameter. For a parameterized curve $\mathbf{r}(t)$ with time $t$, by the chain rule for finding the derivatives of composite functions, we get

$$
\frac{d \mathbf{r}(t)}{d t}=\frac{d \mathbf{r}(s)}{d s} \frac{d s}{d t}=\frac{d \mathbf{r}(s)}{d s} \dot{s}
$$

Also, by the definition of the arc differentiation,

$$
d s=\left|\mathbf{r}^{\prime}(t)\right| d t=\sqrt{\left(\mathbf{r}_{1}^{\prime}(t)\right)^{2}+\left(\mathbf{r}_{2}^{\prime}(t)\right)^{2}+\left(\mathbf{r}_{3}^{\prime}(t)\right)^{2}} d t .
$$

If the frame of the target curve rotates with an extra angular velocity $\left.\omega_{d}\right|_{F}$, then the angular velocity of $\{\mathscr{F}\}$ around inertial frame $\{\mathscr{I}\}$ resolved in frame $\{\mathscr{F}\}$ is defined by

$$
\begin{aligned}
& \left.\boldsymbol{\omega}_{F 2 I}\right|_{F}=\left.\boldsymbol{\omega}_{d}\right|_{F}+\boldsymbol{\omega}_{\text {Darboux } \mid \text { Bishop }} \\
& \quad=\left[\begin{array}{lll}
\left.\boldsymbol{\omega}_{d x}\right|_{F}-k_{2}(s) \dot{\boldsymbol{s}}+\left.\boldsymbol{\omega}_{d y}\right|_{F} & \left.k_{1}(s) \dot{\boldsymbol{s}}+\left.\boldsymbol{\omega}_{d z}\right|_{F}\right]^{\mathrm{T}} .
\end{array}\right.
\end{aligned}
$$

The translational speed vector of $\{\mathscr{W}\}$ towards $\{\mathscr{I}\}$ resolved in $\{\mathscr{I}\}$ is $\left.\mathbf{v}_{W}\right|_{I}=\left[\begin{array}{lll}\dot{x} & \dot{y} & \dot{z}\end{array}\right]=\left.\mathbf{R}_{W 2 I} \mathbf{v}_{W}\right|_{W}$, where $\mathbf{R}_{W 2 I}$ is the rotation matrix from $\{\mathscr{W}\}$ to $\{\mathscr{I}\}$. From Figure 2, the position vector of the UAV resolved in $\{\mathscr{F}\}$ can be presented as

$$
\mathbf{p}_{I}=\mathbf{p}_{d}+\mathbf{R}_{F 2 I} \mathbf{p}_{F} .
$$

According to the rules of finding derivatives of speed vectors in different frames in the kinematics of rigid bodies, we can find the derivative of (19)

$$
\dot{\mathbf{p}}_{I}=\dot{\mathbf{p}}_{d}+\mathbf{R}_{F 2 I} \dot{\mathbf{p}}_{F}+\mathbf{R}_{F 2 I}\left(\left.\boldsymbol{\omega}_{F 2 I}\right|_{F}\right)^{\wedge} \mathbf{p}_{F}
$$

where ()$^{\wedge}$ is the so-called hat mapping, which means, for any two vector, $\mathbf{a}$ and $\mathbf{b},(\mathbf{a})^{\wedge} \mathbf{b}=\mathbf{a} \times \mathbf{b}$ is satisfied. Particularly, for a 3-dimension case, the form of a skew-symmetric matrix of vector $\mathbf{a}=\left(a_{1}, a_{2}, a_{3}\right)^{\mathrm{T}}$ in accordance with a hat mapping is presented as

$$
(\mathbf{a})^{\wedge}=\left[\begin{array}{ccc}
0 & -a_{3} & a_{2} \\
a_{3} & 0 & -a_{1} \\
-a_{2} & a_{1} & 0
\end{array}\right] .
$$

Then we state that $(\mathbf{a})^{\wedge}$ belongs to $\operatorname{so}(3)$. $\operatorname{so}(3)$ is the Lie algebra of 3-dimensional special orthogonal group $\mathrm{SO}(3)$. By premultiplying both sides of (20) with $\mathbf{R}_{I 2 F}$, we get

$$
\mathbf{R}_{I 2 F} \dot{\mathbf{p}}=\mathbf{R}_{I 2 F} \dot{\mathbf{p}}_{d}+\dot{\mathbf{p}}_{F}+\left(\left.\omega_{F 2 I}\right|_{F}\right)^{\wedge} \mathbf{p}_{F} .
$$

Of (22) $\mathbf{R}_{I 2 F} \dot{\mathbf{p}}_{d}$ represents the speed of a point in the target curve towards the frame $\{\mathscr{I}\}$ and is resolved in the frame $\{\mathscr{F}\}$. It is a composition of two parts. The first is the speed resolved in frame $\{\mathscr{F}\}$, that is, $\left.\mathbf{v}_{F}\right|_{F}=\left(\begin{array}{lll}\dot{s} & 0 & 0\end{array}\right)^{\mathrm{T}}$. The second is the speed from the frame $\{\mathscr{F}\}$ towards $\{\mathscr{I}\}$, which is also resolved in $\{\mathscr{F}\}$ denoted as $\left.\mathbf{v}_{F 2 I}\right|_{F}$. To get an analytic expression of $\mathbf{R}_{I 2 F} \dot{\mathbf{p}}_{d}$, some knowledge about theoretical mechanics is necessary to be introduced. When a rigid body makes a general motion which is a combination of translation and rotation, the speed of any point $\mathbf{P}$ in the rigid body can be formulated as

$$
\mathbf{v}=\mathbf{v}_{c}+\boldsymbol{\omega} \times \mathbf{r},
$$

where $\mathbf{v}_{c}$ is the speed of the basic point and $\mathbf{r}$ is the position vector from the basic point to the point $\mathbf{P}$. It is arbitrary to choose the position of the basic point, for $\boldsymbol{\omega}$ never changes with the basic point.

Assume there is an initial position $\mathbf{P}_{0}$ moving with a speed of $\dot{\mathbf{P}}_{0}=\mathbf{v}_{d}$ and rotating with an angular velocity of $\mathbf{P}_{d}$. Then the point $\mathbf{P}_{0}$ moves with the target curve meanwhile the relative position between $\mathbf{P}_{0}$ and the point of the target curve remains unchanged. By (23), one has

$$
\left.\mathbf{v}_{F 2 I}\right|_{F}=\mathbf{R}_{I 2 F}\left(\mathbf{v}_{d}+\left(\omega_{d}\right)^{\wedge}\left(\mathbf{p}_{d}-\mathbf{p}_{0}\right)\right) .
$$

Thus

$$
\begin{aligned}
\mathbf{R}_{I 2 F} \dot{\mathbf{p}}_{d} & =\left.\mathbf{v}_{F}\right|_{F}+\left.\mathbf{v}_{F 2 I}\right|_{F} \\
& =\left.\mathbf{v}_{F}\right|_{F}+\mathbf{R}_{I 2 F}\left(\mathbf{v}_{d}+\left(\omega_{d}\right)^{\wedge}\left(\mathbf{p}_{d}-\mathbf{p}_{0}\right)\right) .
\end{aligned}
$$

On the other hand, the left side of (22) is expressed as

$$
\mathbf{R}_{I 2 F} \dot{\mathbf{p}}_{I}=\left.\mathbf{R}_{W 2 F} \mathbf{v}_{W 2 I}\right|_{W} .
$$

With regard to (25) and (26), (22) can be rewritten as

$$
\begin{aligned}
\dot{\mathbf{p}}_{F}= & \left.\mathbf{R}_{W 2 F} \mathbf{v}_{W 2 I}\right|_{W}-\left.\mathbf{v}_{F}\right|_{F} \\
& -\mathbf{R}_{I 2 F}\left(\mathbf{v}_{d}+\left(\omega_{d}\right)^{\wedge}\left(\mathbf{p}_{d}-\mathbf{p}_{0}\right)\right)-\left(\left.\boldsymbol{\omega}_{F 2 I}\right|_{F}\right)^{\wedge} \mathbf{p}_{F} .
\end{aligned}
$$

Let $\mathbf{R}_{B 2 N}$ be the rotation matrix from the body frame $\{\mathscr{B}\}$ to a navigation frame $\{\mathscr{N}\} . \Omega_{b}=\left[\omega_{x}, \omega_{y}, \omega_{z}\right]^{\mathrm{T}}$ represents the angular velocity of $\{\mathscr{B}\}$ around the frame $\{\widetilde{\mathcal{N}}\}$ resolved in $\{\mathscr{B}\}$. According to the general rule of finding the derivatives of the rotation matrices, the derivative of $\mathbf{R}_{B 2 N}$ with respect to time is

$$
\dot{\mathbf{R}}_{B 2 N}=\mathbf{R}_{B 2 N} \Omega_{b}^{\wedge} .
$$

Since in most time the navigation frame $\{\widetilde{\mathcal{N}}\}$ is considered as an inertial frame, the conclusion is still suitable for the UAV systems. Also, the angular velocity from $\{\mathscr{F}\}$ to $\{\mathscr{W}\}$ resolved in $\{\mathscr{W}\}$ is

$$
\left.\boldsymbol{\omega}_{W 2 F}\right|_{W}=\left.\boldsymbol{\omega}_{W 2 I}\right|_{W}-\left.\boldsymbol{\omega}_{F 2 I}\right|_{W} \cdot
$$

So one has

$$
\dot{\mathbf{R}}_{W 2 F}=\mathbf{R}_{W 2 F}\left(\left.\boldsymbol{\omega}_{W 2 F}\right|_{W}\right)^{\wedge} .
$$

Then (27) and (30) construct the dynamic error equations of the moving frame tracking method. 


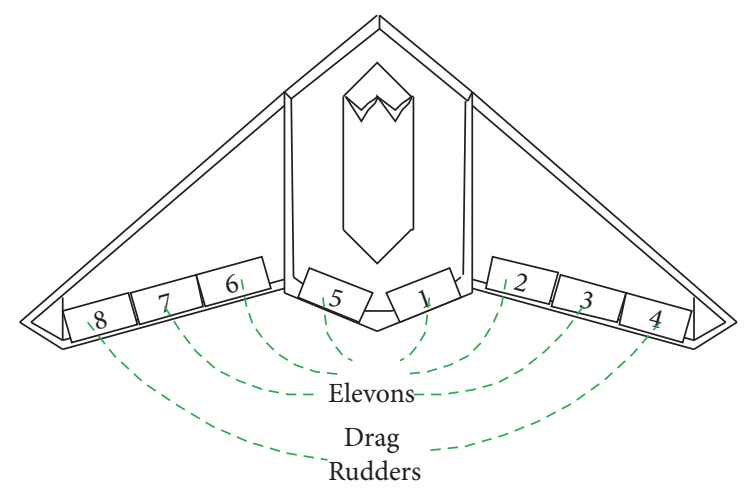

FIGURE 2: The planform and the actuators distribution of the flyingwing UAV.

\section{Simulation}

3.1. Structure of the System. The mass of the UAV, which is used in the simulation, is $2300 \mathrm{~kg}$ and its wingspan equals $8.76 \mathrm{~m}$ with a typical flying-wing configuration. This kind of UAV has no vertical or horizontal tails, so its aerodynamic characteristic is unordinary in various aspects. That leads to a worse static heading stability. The actuators of a flyingwing UAV are not the conventional elevators, ailerons, or rudders. Thus the allocation has to be made to transform the signals of actuators of a flying-wing UAV into the signals of the virtually ordinary actuators, such as $\delta_{e}, \delta_{a}$, and $\delta_{r}$, so that the universality of the designing methodology devised in this paper is guaranteed. Figure 2 shows a planform and the actuators distribution of a flying-wing UAV.

Figure 3 shows the structure of the devised UAV flight control systems in the simulation. The blocks of the target curve, tracking errors of the moving frame, reverse calculation, the UAV model, and the controllers of the innerloops are all included, which can be seen from Figure 3.

A UAV nonlinear state-space system can be obtained with state variables defined by $\mathbf{X}^{\mathrm{T}}=\left[u, v, w, \phi, \theta, \psi, p, q, r, x_{g}\right.$, $\left.y_{g}, h_{g}\right]$ and control input vector $\mathrm{U}^{\mathrm{T}}=\left[\delta_{e}, \delta_{\alpha}, \delta_{r}, \delta_{T}\right]$. According to the traditional definition of a flight control system, $u$, $v$, and $w$ are speed components of body frame, $\theta, \psi$, and $\phi$ represent pitch angle, yaw angle, and bank angle, respectively, $p, q$, and $r$ are angular velocity from body frame to inertial frame resolved in body frame, $x_{g}, y_{g}$, and $h_{g}$ represent the positions of the aircraft in the ground frame which is almost the same as the inertial frame, $V$ is true airspeed, and $\gamma$ and $\varphi$ are flight-path angles between the first/second axis of wind frame and inertial frame, respectively.

Particularly, the controllers of the attitude and trajectory loops are all the ordinary controllers for simplicity. Also when a better tracking result is obtained, the success can be clearly attributed to the devised method rather than the other advanced and complex controllers of the innerloops.

3.2. Moving Frame of an Elliptic Cylinder Helix. The target curve is chosen as an elliptic cylinder helix extending along the horizontal direction. The expression of the target curve with regard to a time parameter is defined as

$$
\mathbf{r}(t)=\left(a_{1} t+c_{1}, a_{2} \cos \left(b_{1} t\right)+c_{2}, a_{3} \sin \left(b_{2} t\right)+c_{3}\right)^{\mathrm{T}},
$$

where $a_{1}, a_{2}, a_{3}, b_{1}, b_{2}, c_{1}, c_{2}$, and $c_{3}$ are all coefficients. By finding the first-order derivative of it with regard to time we have

$$
\mathbf{r}^{\prime}(t)=\left(a_{1},-a_{2} b_{1} \sin b_{1} t, a_{3} b_{2} \cos b_{2} t\right)^{\mathrm{T}} .
$$

Its magnitude is

$$
\left|\mathbf{r}^{\prime}(t)\right|=\sqrt{a_{1}^{2}+\left(-a_{2} b_{1} \sin \left(b_{1} t\right)\right)^{2}+\left(a_{3} b_{2} \cos \left(b_{2} t\right)\right)^{2}} .
$$

By the definition of the Frenet frame and (32), one has

$$
\begin{aligned}
\mathbf{T}(t) & =\frac{\mathbf{r}^{\prime}(t)}{\left|\mathbf{r}^{\prime}(t)\right|} \\
& =\frac{\left(a_{1},-a_{2} b_{1} \sin b_{1} t, a_{3} b_{2} \cos b_{2} t\right)^{\mathrm{T}}}{\sqrt{a_{1}^{2}+\left(-a_{2} b_{1} \sin \left(b_{1} t\right)\right)^{2}+\left(a_{3} b_{2} \cos \left(b_{2} t\right)\right)^{2}}} \\
\frac{d \mathbf{T}}{d s} & =\frac{d \mathbf{T}(t)}{d t} \frac{d t}{d s}=\frac{d \mathbf{T}(t)}{d t} \frac{1}{\left|\mathbf{r}^{\prime}(t)\right|} \\
& =\frac{1}{\left|\mathbf{r}^{\prime}(t)\right|} \frac{d}{d t}\left(\frac{\mathbf{r}^{\prime}(t)}{\left|\mathbf{r}^{\prime}(t)\right|}\right) \\
& =\frac{1}{\left|\mathbf{r}^{\prime}(t)\right|}\left(\frac{\mathbf{r}^{\prime \prime}(t)\left|\mathbf{r}^{\prime}(t)\right|-\mathbf{r}^{\prime}(t)\left(\left|\mathbf{r}^{\prime}(t)\right|\right)^{\prime}}{\left|\mathbf{r}^{\prime}(t)\right|^{2}}\right)
\end{aligned}
$$

For the simplicity of the expression, denote $m=a_{1}{ }^{2}+\left(a_{2} b_{1}\right)^{2}$, $n=\left(a_{3} b_{2}\right)^{2}-\left(a_{2} b_{1}\right)^{2}$, and den $=\left|\mathbf{r}^{\prime}(t)\right|$. Let $b_{1}=b_{2}$; we have

$$
\begin{aligned}
\operatorname{den} & =\left|\mathbf{r}^{\prime}(t)\right|=\sqrt{a_{1}^{2}+\left(-a_{2} b_{1} \sin \left(b_{1} t\right)\right)^{2}+\left(a_{3} b_{2} \cos \left(b_{2} t\right)\right)^{2}}=\sqrt{a_{1}^{2}+\left(a_{2} b_{1}\right)^{2}+\left(\left(a_{3} b_{2}\right)^{2}-\left(a_{2} b_{1}\right)^{2}\right) \cos ^{2}\left(b_{1} t\right)} \\
& =\sqrt{m+n \cos ^{2}\left(b_{1} t\right)}, \\
\operatorname{den}^{\prime} & =\frac{-n \cdot b_{1} \cdot \sin \left(b_{1} t\right) \cdot \cos \left(b_{1} t\right)}{\operatorname{den}}, \\
\frac{d \mathbf{T}(t)}{d t} & =\frac{\left(-a_{1} \operatorname{den}^{\prime},-a_{2} b_{1}^{2} \cos \left(b_{1} t\right) \cdot \operatorname{den}+a_{2} b_{1} \sin \left(b_{1} t\right) \cdot \operatorname{den}^{\prime},-a_{3} b_{2}^{2} \sin \left(b_{2} t\right) \cdot \operatorname{den}-a_{3} b_{2} \cos \left(b_{2} t\right) \cdot \operatorname{den}^{\prime}\right)^{\mathrm{T}}}{\operatorname{den}^{2}} .
\end{aligned}
$$




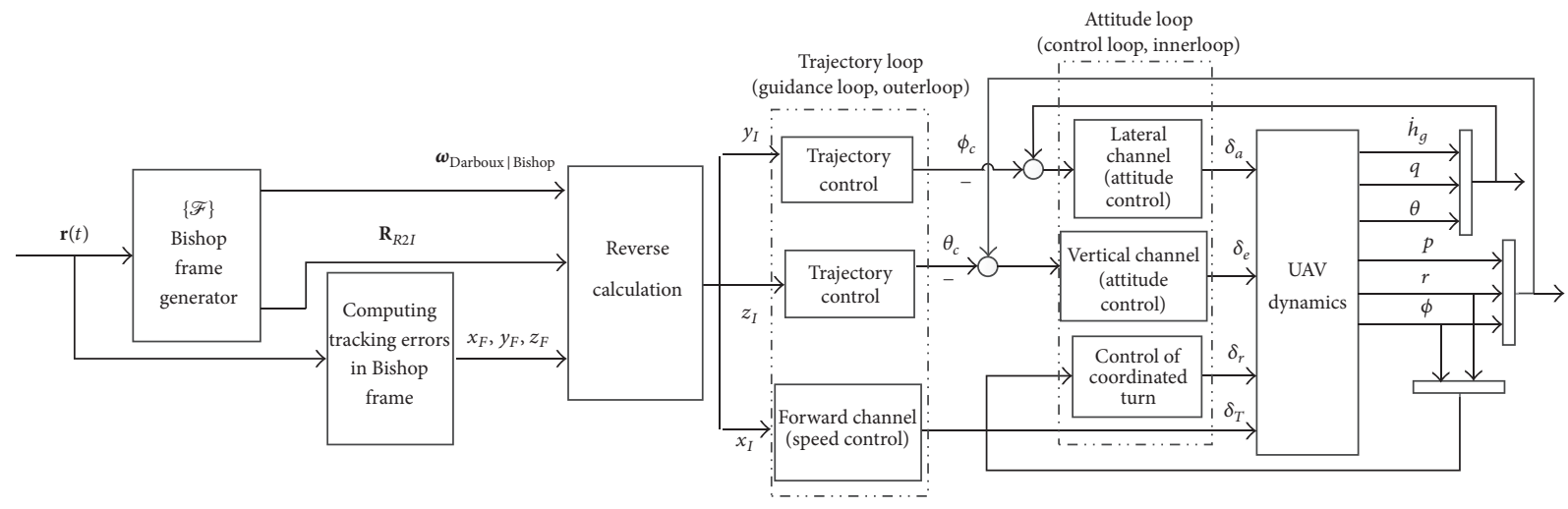

FIgURE 3: Simulation structure of the UAV flight control system.

By (34) and (35)

$$
\begin{aligned}
\frac{d \mathbf{T}}{d s} & =\frac{d \mathbf{T}(t)}{d t} \frac{1}{\left|\mathbf{r}^{\prime}(t)\right|} \\
& =\frac{\left(-a_{1} \operatorname{den}^{\prime},-a_{2} b_{1}^{2} \cos \left(b_{1} t\right) \cdot \operatorname{den}+a_{2} b_{1} \sin \left(b_{1} t\right) \cdot \operatorname{den}^{\prime},-a_{3} b_{2}^{2} \sin \left(b_{2} t\right) \cdot \operatorname{den}-a_{3} b_{2} \cos \left(b_{2} t\right) \cdot \operatorname{den}^{\prime}\right)^{\mathrm{T}}}{\operatorname{den}^{2}\left|\mathbf{r}^{\prime}(t)\right|}
\end{aligned}
$$

Then $\mathbf{N}(t)$ and $\mathbf{B}(t)$ are obtained

$$
\begin{aligned}
& \mathbf{N}(t)=\frac{d \mathbf{T}}{d s}\left|\frac{d \mathbf{T}}{d s}\right|^{-1}, \\
& \mathbf{B}(t)=\mathbf{T}(t) \times \mathbf{N}(t) .
\end{aligned}
$$

According to $(4) \sim(5)$, that is, the transformation rule of the Frenet frame and the Bishop-1 frame, one has

$$
\begin{aligned}
\mathbf{T} & =\mathbf{T}, \\
\mathbf{N}_{1} & =\cos \theta_{F} \mathbf{N}-\sin \theta_{F} \mathbf{B}, \\
\mathbf{N}_{2} & =\sin \theta_{F} \mathbf{N}+\cos \theta_{F} \mathbf{B}, \\
\theta_{F}(t) & =\int \tau(s) d s+\theta_{0}=\int \tau(t)|r(t)|^{\prime} d t+\theta_{0}, \\
\tan \theta_{F} & =\frac{k_{2}}{k_{1}} \\
\kappa & =\sqrt{k_{1}^{2}+k_{2}^{2}}=\sqrt{k_{1}^{2}+k_{1}^{2} \tan ^{2} \theta_{F}} \\
& =\sqrt{1+\tan ^{2} \theta_{F}}\left|k_{1}\right| .
\end{aligned}
$$

Thus

$$
\begin{aligned}
& k_{1}= \pm \frac{\kappa}{\sqrt{1+\tan ^{2} \theta_{F}}} \\
& k_{2}=\tan \theta_{F} \cdot k_{1} .
\end{aligned}
$$

By (2), the expressions of the Frenet curvature and torsion, we get

$$
\begin{aligned}
& \kappa=\left|\frac{d \mathbf{T}}{d s}\right|=\left|\frac{d \mathbf{T}}{d t} \frac{d t}{d s}\right|=\left.\left|\mathbf{T}^{\prime}(t)\right| r^{\prime}(t)\right|^{-1} \mid \\
& \tau=-\frac{d \mathbf{B}}{d s} \cdot \mathbf{N}=-\frac{d t}{d s} \frac{d \mathbf{B}}{d t} \cdot \mathbf{N}=-\left|r^{\prime}(t)\right|^{-1} \frac{d \mathbf{B}}{d t} \cdot \mathbf{N} .
\end{aligned}
$$

By (31), (32), (34), (35), (38), and (39), the Bishop frame of the target curve $\mathbf{r}(t)$ used in the simulation is formulated. For other complex curves of $3 \mathrm{D}$ trajectory, this methodology is still feasible as long as their analytic expressions are available (either formulated by their definitions or curve fitting methods) with regard to the time parameter $t$. Actually if a curve is fitted by a linear or quadratic function, the computation of its moving frame may be easier as its higher derivatives equal zero with orders being more than 3 .

In simulation, according to (31), the values of the coefficients are chosen by

$$
\begin{aligned}
& a_{1}=200, \\
& a_{2}=300, \\
& a_{3}=-250, \\
& b_{1}=0.1,
\end{aligned}
$$




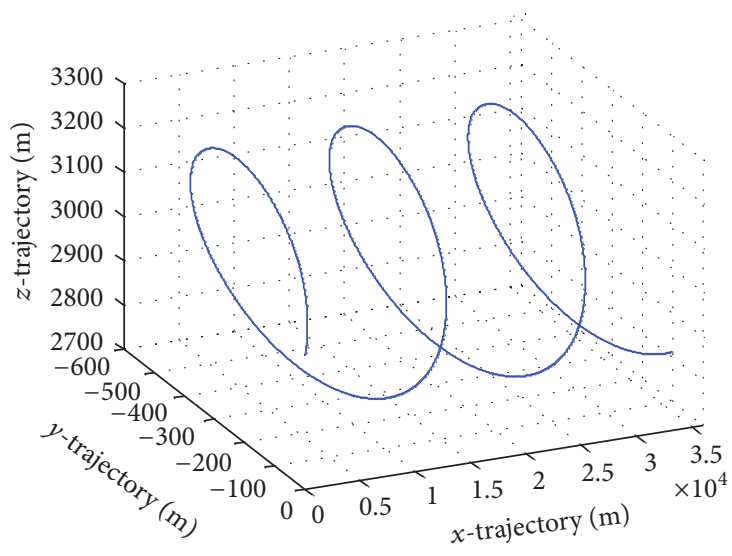

(a)

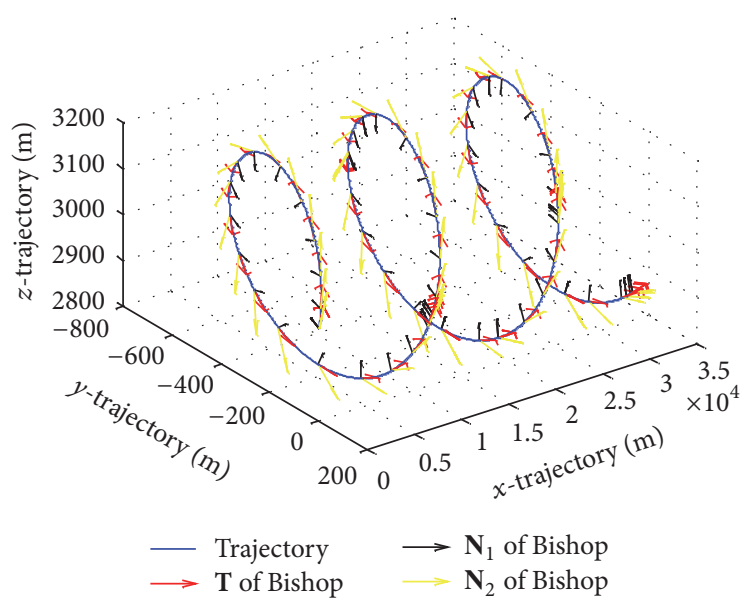

(c)

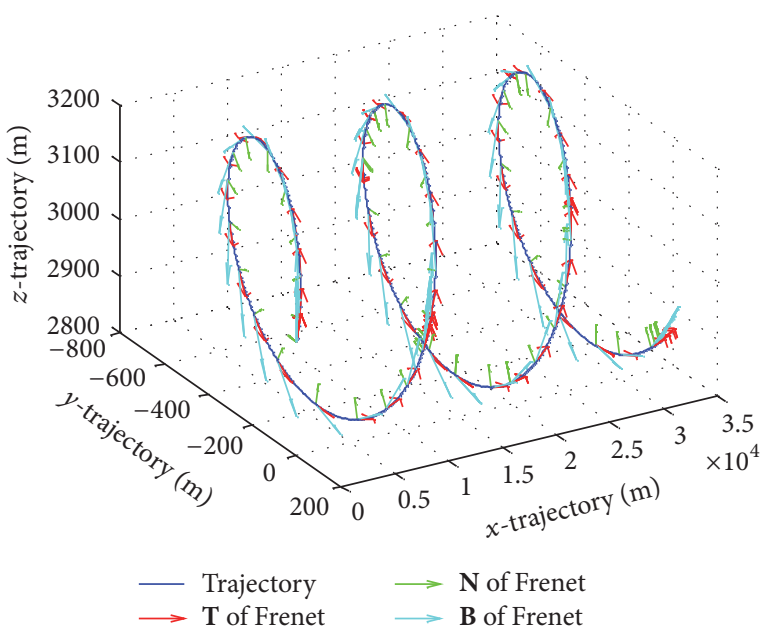

(b)

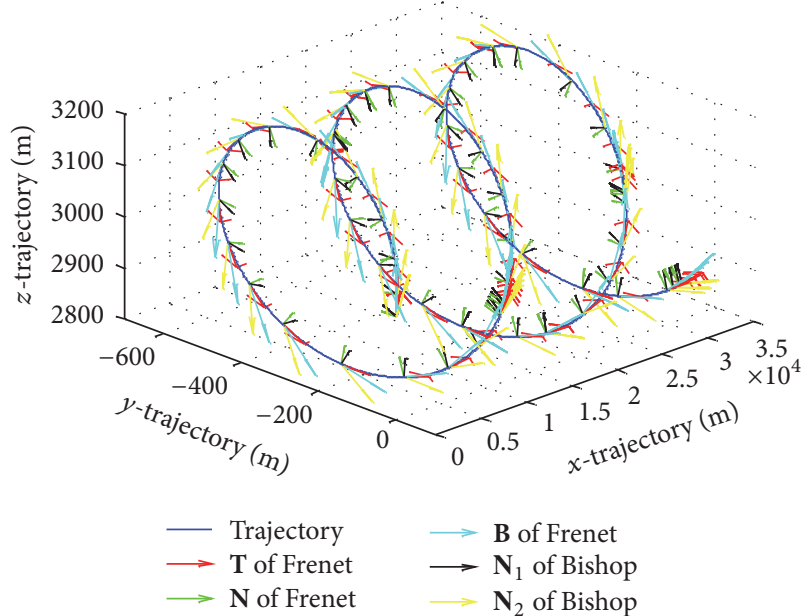

(d)

Figure 4: (a) Trajectory of target curve, (b) the target curve and its Frenet frame, (c) the target curve and its Bishop frame, and (d) the target curve and its moving frames.

$$
\begin{aligned}
& b_{2}=0.1, \\
& c_{1}=0, \\
& c_{2}=-300, \\
& c_{3}=-3000 .
\end{aligned}
$$

The curves of the target curve $\mathbf{r}(t)$ and its moving frames are shown in Figures 4(a)-4(d).

In Figure 4 the target curve is the blue curve which is the target to track. T, N, and $\mathbf{B}$, respectively, represent the tangent vector, principal normal vector, and secondary normal vector and they are the three orthonormal axes of the Frenet frame. Meanwhile $\mathbf{T}, \mathbf{N}_{1}$, and $\mathbf{N}_{2}$ represent the three orthonormal axes of the Bishop frame.

For the lengths of the intervals of three axes of the moving frames are different, there are some zoom in and out of these axes in Figure 4 for the purpose of clear identification. In Figures $4(\mathrm{~b})-4(\mathrm{~d})$ it is easy to identify the tangent vector $\mathbf{T}$ of the curve as the red arrow. The other two axes such as $\mathbf{N}$ and B or $\mathbf{N}_{1}$ and $\mathbf{N}_{2}$ construct the orthonormal coordinate frames with T. In Figures 4(c)-4(d) the initial parameter $\theta_{F}$ of the Bishop frame is chosen as $50^{\circ}$. According to the definitions of the two moving frames, there is a superposition of them when that parameter equals zero.

3.3. Tracking Result. The beginning of the tracking action starts from the moment the UAV arrives in a small neighborhood of an arbitrary point of the target curve. The initial value of the speed vector of the body frame is chosen at $\mathbf{V}_{b 0}=[200,0,-2]$ and the attitude angles vector is $\phi, \theta,\left.\psi\right|_{0}=$ $[0,2,0]$, where the units are, respectively, " $\mathrm{m} / \mathrm{s}$ " and “" . The high speed is needed to provide enough lift. Also a larger drag force, which is proportional to the square of the airspeed, is needed to keep the maneuverability in the forward channel. The opening range of the throttle is $[0,1]$, which means the throttle cannot provide a force in the opposite direction. So the total force of the forward channel 


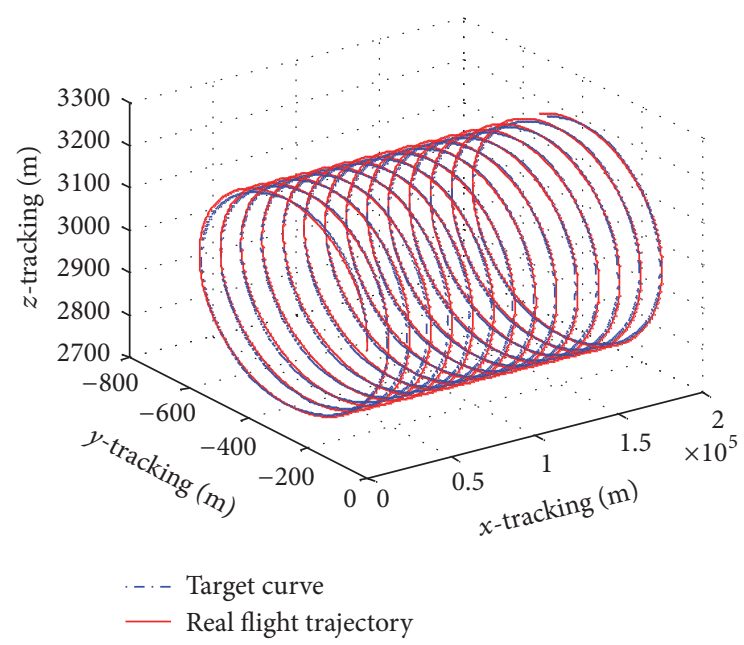

(a)

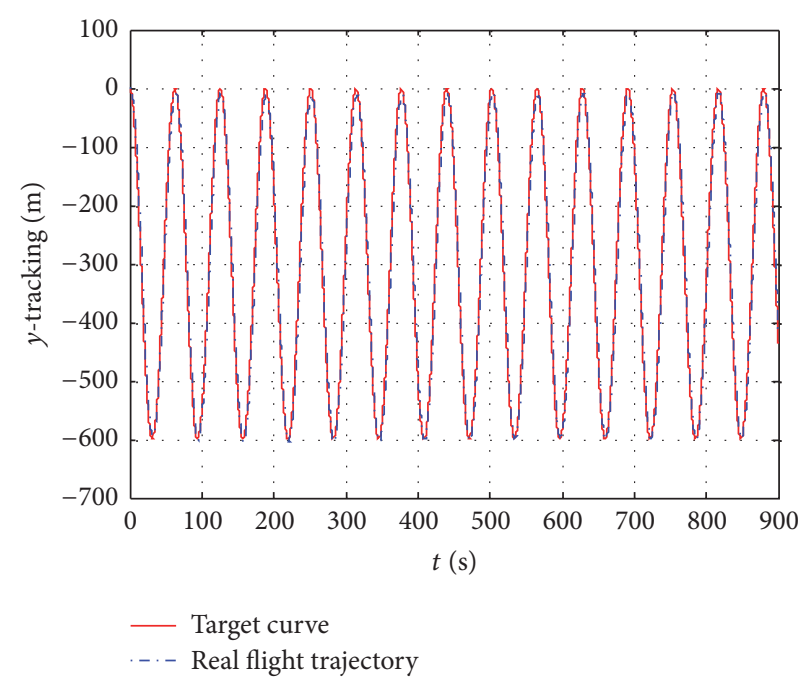

(c)

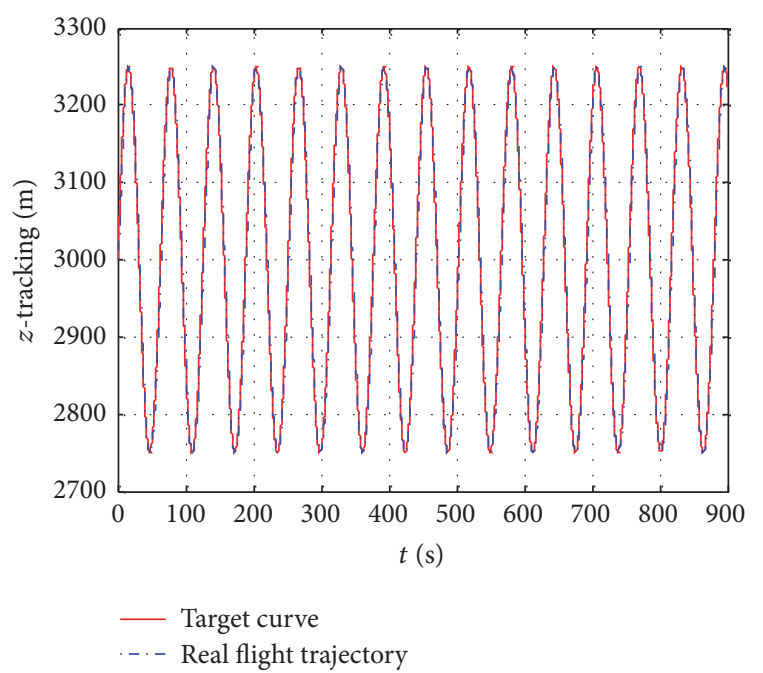

(b)

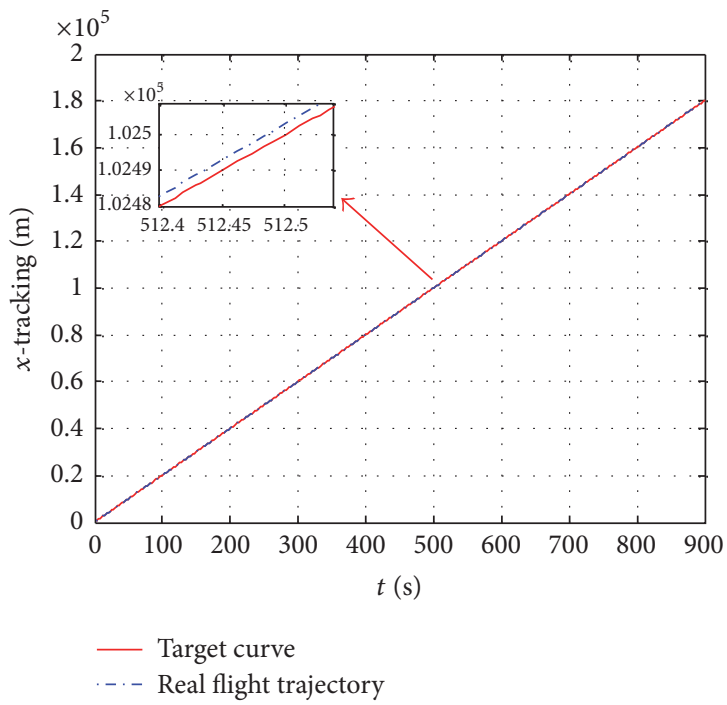

(d)

FIGURE 5: (a) 3D trajectory tracking result, (b) tracking curves in the vertical direction, (c) tracking curves in the lateral direction, and (d) tracking curves in the forward direction.

will not change its direction if the drag is not larger enough and finally it will result in an increasing forward speed. The result of trajectory tracking is shown in Figures 5(a)5(d).

Figure 5(a) shows the 3D trajectory tracking result. From Figure 5(a) we can see that, during the simulation time of $900 \mathrm{~s}$, UAV keeps on following the target curve with hardly any time delay. The mission of trajectory tracking is satisfactorily completed. Figures $5(\mathrm{~b})-5(\mathrm{~d})$, respectively, show the tracking curves with respect to time in the vertical, lateral, and forward directions of the inertial frame. As the airspeed of the UAV is about $200 \mathrm{~m} / \mathrm{s}$, the deviation could be quite large if the tracking method is not good enough. The vertical steady-state tracking error is less than $2 \mathrm{~m}$ within $0.8 \mathrm{~s}$ time delay, the lateral error is less than $7 \mathrm{~m}$ within $1.1 \mathrm{~s}$ time delay, and the forward error is less than $5 \mathrm{~m}$, which are shown in these figures.

\section{Conclusions}

The moving frame tracking method devised in this paper based on the classical differential geometry is interdisciplinary. It is a trial to solve the trajectory tracking problems in a new direction avoiding the complex designing of the innerloop controllers. Although the innerloop controllers are simple, the whole flight control system in the simulation is much more complicated since a 6 DOF model of a large flying-wing UAV is employed. From the simulation it can be seen that a rapid, accurate, and safe trajectory tracking result is obtained.

\section{Competing Interests}

The authors declare that there are no competing interests regarding the publication of this paper. 


\section{References}

[1] R. Curry, M. Lizarraga, B. Mairs, G. Elkaim, and G. H. Elkaim, " $L_{2}^{+}$an improved line of sight guidance law for UAVs," in Proceedings of the American Control Conference (ACC '13), June 2013.

[2] J. J. Deyst, J. P. How, and S. Park, "Lyapunov stability of a nonlinear guidance law for UAVs," in Proceedings of the AIAA Atmospheric Flight Mechanics Conference, pp. 1192-1208, August 2005.

[3] H. Chen, K.-C. Chang, and C. S. Agate, "Tracking with UAV using tangent-plus-Lyapunov vector field guidance," in Proceedings of the 12th International Conference on Information Fusion (FUSION '09), pp. 363-372, Seattle, Wash, USA, July 2009.

[4] A. M. Shinji, "Lateral guidance control of UAV using feedback error learning," in Proceedings of the AIAA Infotech@Aerospace 2007 Conference and Exhibit, pp. 2007-2727, May 2007.

[5] S. Park, J. Deyst, and J. P. How, "Performance and Lyapunov stability of a nonlinear path-following guidance method," Journal of Guidance, Control, and Dynamics, vol. 30, no. 6, pp. 1718-1728, 2007.

[6] R. Yanushevsky, Guidance of Unmanned Aerial Vehicles, CRC Press, 2011.

[7] R. L. Bishop, “There is more than one way to frame a curve," The American Mathematical Monthly, vol. 82, pp. 246-251, 1975.

[8] A. J. Hanson and H. Ma, "Parallel transport approach to curve framing," Tech. Rep., Indiana University Compute Science Department, 1995.

[9] D. Carroll, E. Köse, and I. Sterling, "Improving Frenet's frame using Bishop's frame," Journal of Mathematics Research, vol. 5, no. 4, pp. 97-106, 2013.

[10] S. Kiziltug, S. Kaya, and O. Tarakci, “Tube surfaces with type-2 bishop frame of weingarten types in $\mathrm{E}^{3}$," International Journal of Mathematical Analysis, vol. 7, no. 1-4, pp. 9-18, 2013.

[11] Ş. Kiliçoğlu and H. H. Hacisalihoğlu, "On the ruled surfaces whose frame is the Bishop frame in the Euclidean 3-space," International Electronic Journal of Geometry, vol. 6, no. 2, pp. 110-117, 2013.

[12] T. Körpınar and E. Turhan, "Biharmonic curves according to parallel transport frame in E4," Boletim da Sociedade Paranaense de Matemática, vol. 31, no. 2, pp. 213-217, 2013.

[13] T. Oliveira, P. Encarnacao, and A. P. Aguiar, "Moving path following for autonomous robotic vehicles," in Proceedings of the 12th European Control Conference (ECC '13), pp. 3320-3325, Zurich, Switzerland, July 2013.

[14] I. Kaminer, O. Yakimenko, A. Pascoal, and R. Ghabcheloo, "Path generation, path following and coordinated control for time-critical missions of multiple UAVs," in Proceedings of the American Control Conference, pp. 4906-4913, Minneapolis, Minn, USA, June 2006.

[15] V. Cichella, V. Dobrokhodov, I. Kaminer, L. Marconi, and R. Naldi, "On 3D path following control of a ducted-fan UAV on $\mathrm{SO}(3)$," in Proceedings of the 50th IEEE Conference on Decision and Control and European Control Conference (CDC-ECC '11), pp. 3578-3583, IEEE, Orlando, Fla, USA, December 2011.

[16] V. Cichella, E. Xargay, V. Dobrokhodov, I. Kaminer, A. M. Pascoal, and N. Hovakimyan, "Geometric 3D path-following control for a fixed-wing UAV on $\mathrm{SO}(3)$," in Proceedings of the AIAA Guidance, Navigation and Control Conference, Portland, Ore, USA, August 2011.
[17] T. Lee, M. Leok, and N. McClamroch, "Geometric tracking control of a quadrotor UAV on SE(3)," https://arxiv.org/abs/1003 $.2005 \mathrm{vl}$.

[18] T. Lee, "Robust global exponential attitude tracking controls on $\mathrm{SO}(3)$," in Proceedings of the 1st American Control Conference (ACC '13), pp. 2103-2108, June 2013.

[19] T. Lee, "Geometric tracking control of the attitude dynamics of a rigid body on SO(3)," in Proceedings of the American Control Conference, pp. 1200-1205, San Francisco, Calif, USA, 2011. 


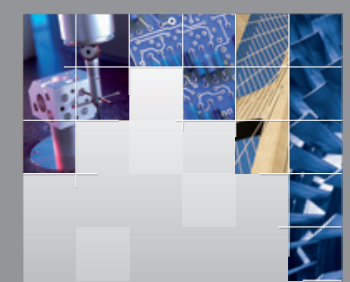

\section{Enfincering}
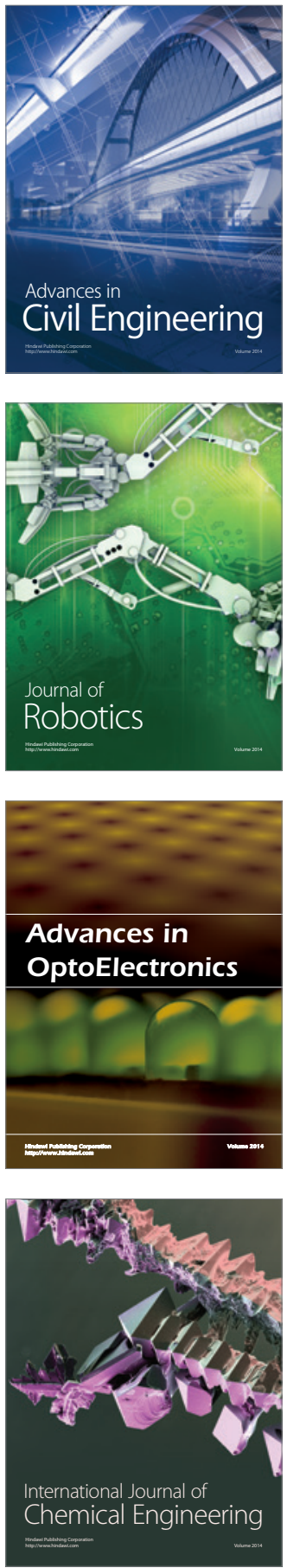

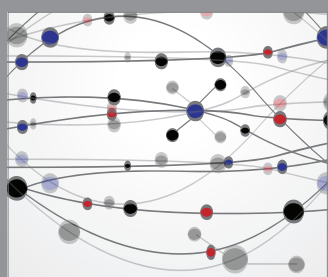

The Scientific World Journal

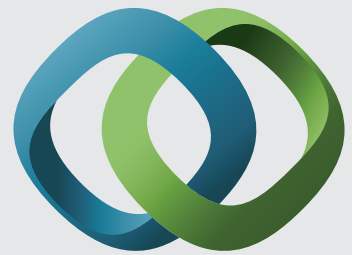

\section{Hindawi}

Submit your manuscripts at

http://www.hindawi.com
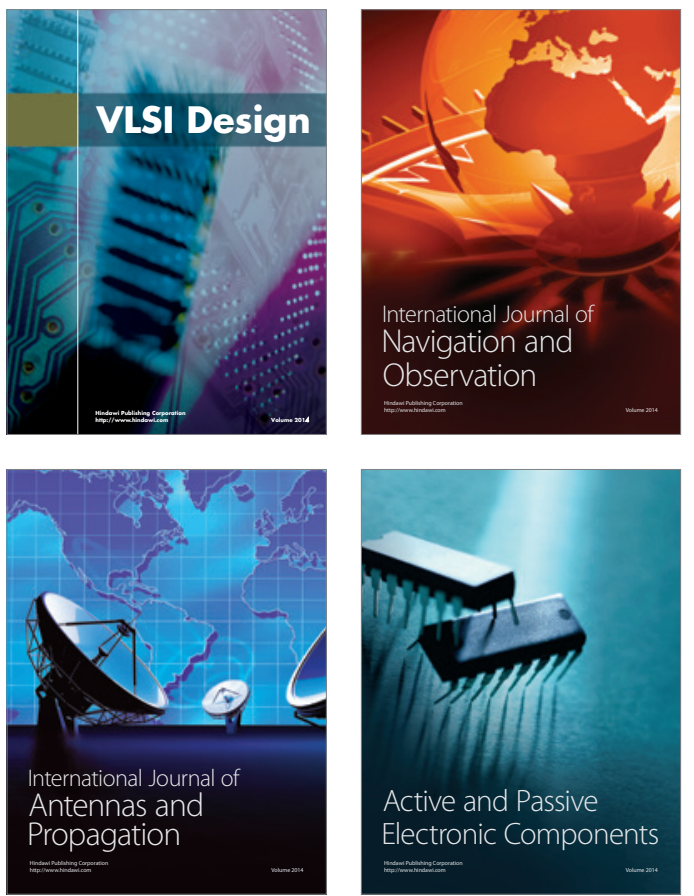
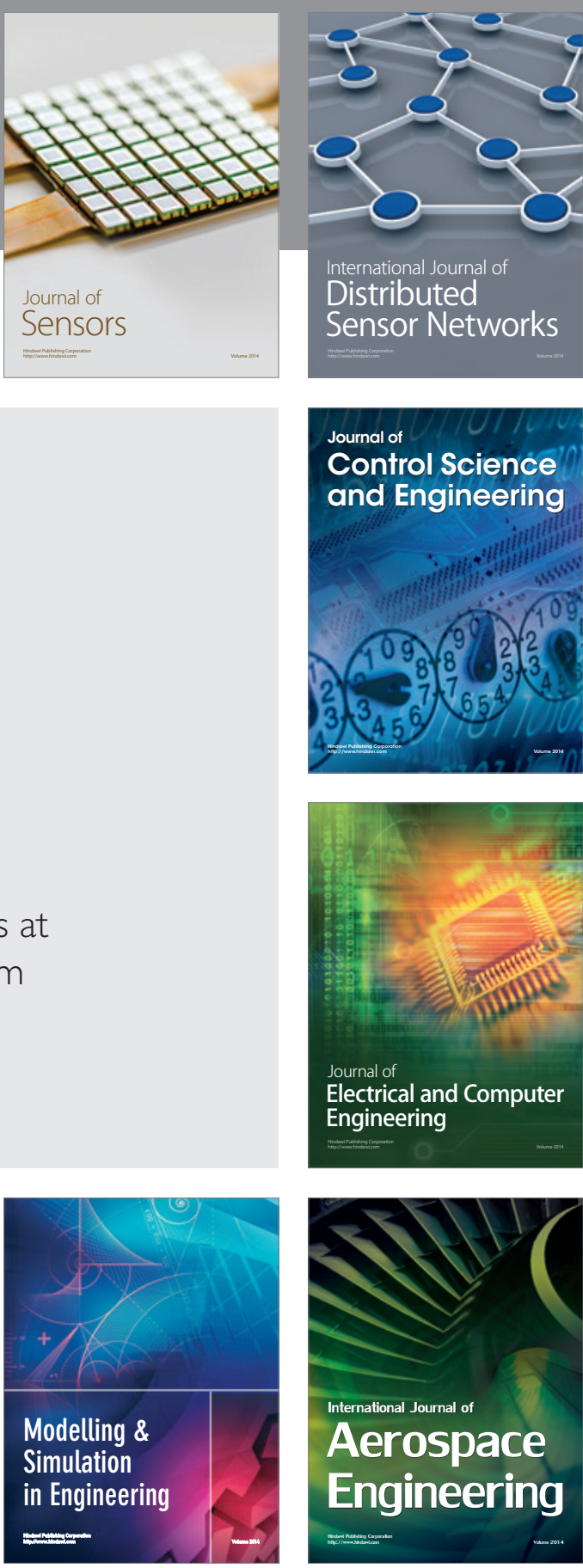

International Journal of

Distributed

Sensor Networks

Journal of

Control Science

and Engineering
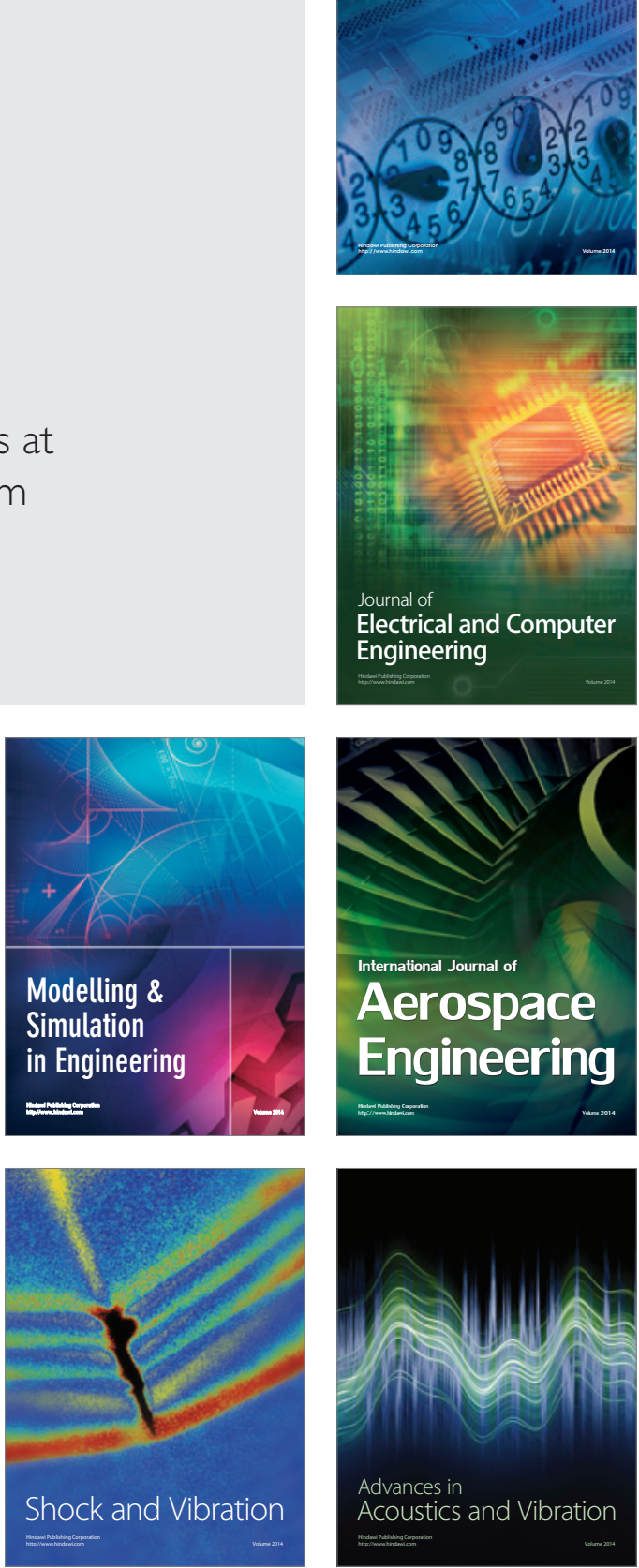